\title{
Pulmonary Alveolar Microlithiasis and Pregnancy - A Case Report
}

\section{MUKTA AGARWAL}

AllMS: All India Institute of Medical Sciences

RITU SINGH ( $\nabla$ ritu.singh@aiimspatna.org )

All India Institute of Medical Sciences - Patna

\section{Sudwita Sinha}

AllMS: All India Institute of Medical Sciences

Neeraj Kumar

AllMS: All India Institute of Medical Sciences

\section{Research Article}

Keywords: Pulmonary alveolar microlithiasis( PAM) , Pregnancy with PAM

Posted Date: June 14th, 2021

DOI: https://doi.org/10.21203/rs.3.rs-586312/v1

License: (c) (1) This work is licensed under a Creative Commons Attribution 4.0 International License. Read Full License 


\section{Abstract}

Pulmonary alveolar microlithiasis (PAM) is a rare autosomal recessive disease with a high penetrance characterized by widespread intra-alveolar accumulation of innumerable minute calculi called microliths. Pregnancy with PAM is even rarer. Here we are presenting the second case in India of 28yrs G2P1L1 37 weeks 1 day, known case of PAM with gestational hypothyroidism. She had difficulty in breathing which has increased from NYHA- grade II to NYHA- grade III from the fifth month of gestation. She had parrot beak (grade 3) clubbing. She was having intrauterine growth restriction with a growth discrepancy of four weeks. The patient was taken for cesarean section in view of a breech with premature rupture of the membrane with intrauterine growth restriction. Since lung function is compromised, oxygen supply to the baby is expected to be compromised, which can lead to intrauterine growth restriction, which is evident in this patient in both of her pregnancies.

\section{Introduction}

Pulmonary alveolar microlithiasis (PAM) is a rare autosomal recessive disease with a high penetrance characterized by widespread intra-alveolar accumulation of innumerable minute calculi called microliths. [1]

PAM presents most commonly in the second and third decade of life but may be seen at any age. [1]Many patients will develop the progressive disease with restrictive lung function impairment, dyspnoea and cough, hypoxia and respiratory failure, secondary pulmonary hypertension, and ultimately death. [2]

PAM affects both sexes with a slight predominance in males (male/female ratio 0.56) [1]

Pregnancy with PAM is very rare. Very few cases are reported till date. Here we are going to present a case of pregnancy with PAM

\section{Case Report}

28yrs G2P1L1 37 weeks 1 day, known case of PAM with gestational hypothyroidism presented in the outpatient department of obstetrics and gynaecology of tertiary care hospital. She had difficulty in breathing which has increased from NYHA-grade II to NYHA - grade III from the fifth month of gestation. In pregnancy, vomiting was associated with cough. She had started pain in the lateral side of the chest in 7-8 months of pregnancy. She was taking treatment from a local obstetrician. General treatment for pregnancy was given, she had not taken any specific treatment for increasing dyspnoea.

In her obstetric history, her first child was 5.5 years old, of $2.2 \mathrm{~kg}$ birth weight was born vaginally in tertiary care hospital, which was uneventful.

The patient was accidentally diagnosed with PAM 8 years back when she had been evaluated for pain in the abdomen. She remained asymptomatic for 3 years then she developed cough with expectorant which 
was more in winter( not typically dry as occurs in PAM ), progressive dyspnoea, swelling in the leg, syncopal attack at emotional stress, for which she was advised tab lasilactone $25 \mathrm{mg}$, syrup levodropropizine 1 tsp TDS, yoga therapy for 1 year. Serial CT scan showed progression of the disease and the patient was advised lung transplantation, but she had never been on oxygen for her symptoms. Her pulmonary function test at the onset of symptoms was FEV1-1.56(50.1\% Pred), FVC1.58(44.4\%Pred), FEV1/FVC- 98.87\%, PEF - 3.50(50.2\%Pred). At the time of presentation to our hospital, moderate restriction (40-45\%) was present. FEV1-1.21L (42.4\%)

There was no history of similar complaints in the family.

On examination her pulse was $94 / \mathrm{min}$, blood pressure was $134 / 86 \mathrm{~mm}$ of $\mathrm{Hg}$. Parrot beak (grade 3 ) clubbing was present.[Image 1]

On per abdomen examination, her fundal height was corresponding to less than 32 weeks of gestation with breech presentation. Her ultrasound showed a fetus with the approximate gestational age of 34 weeks, an effective fetal weight of $2200 \mathrm{gm}$, and an amniotic fluid index of five with an increased umbilical artery S/D ratio of 3.71. Chest x-ray with shield showed bilateral diffuse sand storm appearance more in mid to lower zone. In the CT scan there was diffuse inter and intralobular calcified septal thickening in the bilateral lung without any sparing or gradient, diffuse tiny nodular opacities was seen in the bilateral lung, dense subpleural calcification was seen in the bilateral upper lobe.[Image 2] ECHO showed-Grade I LVDD, Mild TR, Normal LV Systolic function. LVEF- 55-60\%.

Patient was managed by a multidisciplinary approach in pulmonary medicine \& intensivist and was given nebulization with levosalbutamol sulphate and ipratropium bromide $5 \mathrm{ml}$ and inj hydrocortisone $100 \mathrm{mg}$ iv prior to surgery. Caesarean section under spinal anaesthesia was done in view of breech with oligohydramnios explaining the moderate risk of postoperative respiratory failure and chances of mechanical ventilation.

Intraoperative her vitals remained stable throughout with Spo2 95\% at 5 lit of oxygen. A live female baby delivered $2.2 \mathrm{~kg}$ Apgar 6/10, 9 /10. Liqour was less in amount.

Her postnatal period was uneventful. The patient was advised discharge on day four of the operation on oral antibiotics, iron, calcium. The patient was counselled for contraception. The patient preferred vasectomy. The patient is still under follow-up.

\section{Discussion}

Until today, a little fewer than 1100 cases of PAM( including male and female) have been reported since Harbitz published the first case in 1918.[2] Approximately 80 cases were reported from India out of 1022 cases, as Castellana et al reported in 2015.[1]

Pulmonary microlithiasis with pregnancy is rare. On reviewing the literature, six cases of pulmonary microlithiasis with pregnancy have been reported all over the world, first case reported was in 2006 from 
South America.[3]

Out of the total 6 cases, only two are from Asia.

From India, the first case reported was from Cuttack, in 2015. 27 years old female presented in pulmonary medicine department, 1 week after delivery in obstetric ward vaginally.

This is the second case from India of pregnancy with PAM. Very little is known about how PAM and pregnancy affect each other. From pathophysiology it is evident that as lung function is compromised it may cause intrauterine growth restriction, this is reflecting in our case. And in this present case, intrauterine growth restriction is present in both of her pregnancies.

Because of the paucity of data and rarity of the condition, the management protocol for these patients is still not clear, that whether pregnancy should be allowed or medical termination of pregnancy can be an option if the patient presents in early pregnancy. Management should be guided by the patient's present condition, her lung function test \& need for oxygen. These pregnancies should be managed at a tertiary care centre with a multidisciplinary approach involving pulmonologist, intensivist, obstetrician, paediatrician for optimal patient outcome.

\section{Declarations}

i Funding - No funding required.

ii. Conflicts of interest/Competing interests - There is no conflict of interest / competing interest in this case report.

iii. Consent to participate - Consent taken to participate in the case report from the patient, if her name is not disclosed.

iv. Consent for publication - Consent taken for publication in the case report from the patient, if her name is not disclosed.

vi. Availability of data and material (data transparency)

vii. Code availability (software application or custom code)

viii. Authors' contributions- First author, Agarwal $\mathrm{M}$ - unit head, revised the content critically for important intellectual content and final approval of the version to be published.

Second Author, Singh S - Corresponding author, done initial drafting of the content.

Third, Sinha S- Seen a patient for the first time in OPD and admitted the patient.

Fourth Author, Kumar M - Given anaesthesia during surgery and managed patient post operatively. 


\section{References}

1.Castellana G, Castellana G, Gentile M, Castellana R, Resta O. Pulmonary alveolar microlithiasis: Review of the 1022 cases reported worldwide. Eur Respir Rev 2015;24:607-20.

2. Bendstrup E, Jönsson ÅLM. Pulmonary alveolar microlithiasis: no longer in the stone age. ERJ Open Res. 2020 Sep 14;6(3):00289-2020. DOI: 10.1183/23120541.00289-2020. PMID: 32964001; PMCID: PMC7487355.

3 Rodríguez, F., Ferrer, J., Briozzo, L. and Pons, J., 2006. Pulmonary alveolar microlithiasis and pregnancy. The Journal of Maternal-Fetal \& Neonatal Medicine, 19(4), pp.239-241.

4.Sethy HK, Trilochan BP, Panda G, Misra M. Pulmonary alveolar microlithiasis in pregnancy: a rare case report. Journal of Evolution of Medical and Dental Sciences. 2015 Dec 7;4(98):16387-90.Vismara MF, 